\title{
INFLUÊNCIA DA POSIÇÃO E DA PROFUNDIDADE DE SEMEADURA NA EMERGÊNCIA DE PLÂNTULAS DE AÇAÍ (Euterpe oleracea Mart. - ARECACEAE) ${ }^{1}$
}

\author{
BRENO MARQUES DA SILVAE SILVA², FABIOLA VITTI MÔRO ${ }^{3}$, RUBENS SADER $^{4}$, NILCE NAOMI KOBORI $^{5}$
}

RESUMO - O objetivo deste trabalho foi determinar a posição e a profundidade de semeadura mais adequadas para a emergência de plântulas de açaizeiro. As sementes foram colocadas para germinar nas profundidades de 0; 3 e 6 cm e nas seguintes posições: sementes com a rafe perpendicular à superfície do substrato e poro germinativo para cima, rafe perpendicular e poro germinativo para baixo, rafe paralela à superfície e poro germinativo para baixo e rafe paralela à superfície e poro germinativo para cima. A emergência de plântulas de açaizeiro com a rafe perpendicular à superfície do substrato e poro germinativo para cima é a mais adequada, pois proporciona igual porcentagem e menor tempo médio de emergência. Profundidades iguais ou superiores a $3 \mathrm{~cm}$ são inadequadas para semeadura de Euterpe oleracea Mart.

Termos para indexação: açaí, palmeira, plântula, semeadura.

\section{INFLUENCE OF THE POSITION AND THE DEPTH OF SOWING IN THE EMERGENCY OF AÇAI (Euterpe oleracea Mart. - ARECACEAE) SEEDLINGS}

\begin{abstract}
The present work had the objective to determine the most suitable depth and position of sowing for the emergency of açaizeiro seedlings. The seeds were placed to germinate at the depths of $0 ; 3$ and $6 \mathrm{~cm}$ and in the following positions: with the raphe perpendicular to the surface of the substratum and germinative aperture up, the raphe perpendicular and germinative aperture down, the raphe parallel to the low surface and germinative aperture down, and raphe parallel to the surface and germinative aperture up. The emergency of açaizeiro seedlings with rafe perpendicular to the surface of the substratum and germinative aperture up is the most suitable, as it provides an equal percentage and minor average time of emergency. Depths equal or superior to $3 \mathrm{~cm}$ are inadequate for sowing of Euterpe oleracea Mart.
\end{abstract}

Index terms: açai, palm, seedlings, sowing.

Euterpe oleracea Mart., conhecida popularmente por açaí ou açaizeiro, é uma espécie nativa da Amazônia, sendo encontrada em terrenos de várzea, igapós e terra firme, muitas vezes em formações quase puras. No Brasil, o palmito de açaizeiro é amplamente utilizado; porém, na Amazônia, o principal produto são seus frutos, sendo consumidos in natura ou processados na forma de sucos, vinhos, sorvetes, cremes, picolés e licores (Oliveira \& Fernandes, 2001; Donadio et al., 2003).

A semeadura muito profunda dificulta a emergência das plântulas e aumenta o período de suscetibilidade a patógenos (Napier, 1985; Marcos-Filho, 2005). Uma germinação rápida e uniforme das sementes, seguida por imediata emergência das plântulas são características altamente desejáveis na formação de mudas; quanto maiores o tempo de permanência da plântula nos estágios iniciais de desenvolvimento e a demora em emergir do solo, mais vulnerável estará às condições adversas do meio (Martins et al., 1999a; Marcos-Filho, 2005).Por outro lado, semeaduras rasas podem facilitar o ataque de predadores ou danos decorrentes da irrigação ou, ainda, a exposição e a destruição da raiz primária (Jeller \& Perez, 1997).

Para avaliar os efeitos da posição e da profundidade de semeadura na emergência de plântulas de Euterpe oleracea Mart., frutos de Euterpe oleracea Mart. foram coletados em março de 2005, de matrizes selecionadas, da Área de Proteção Ambiental do Curiau, localizada no município de Macapá-AP, Brasil. O beneficiamento foi realizado por meio da imersão em água morna $\left(45^{\circ} \mathrm{C} \pm 5\right)$, durante 15 minutos, e posterior fricção manual em peneira de malha de aço, como recomendado por Cunha \& Jardim (1995). O teor de água do lote de sementes foi determinado por meio da secagem de quatro repetições de 25 sementes em estufa a $105^{\circ} \mathrm{C} \pm 3$, por 24 horas (Brasil, 1992).

Para as avaliações dos efeitos da posição da semente e da profundidade de semeadura na emergência e vigor de plântulas, três repetições de 50 sementes foram colocadas para germinar em areia, nas seguintes posições: sementes com a rafe perpendicular à superfície do substrato e poro germinativo

${ }^{1}$ (Trabalho 019-2006). Recebido em 02-03-2006. Aceito para publicação em 05-12-2006.

Bolsista de Mestrado do CNPq - Mestrando em Agronomia [Produção e Tecnologia de Sementes]. UNESP - Universidade Estadual Paulista "Júlio de Mesquita Filho"/FCAV - Faculdade de Ciências Agrárias e Veterinárias/DPV - Departamento de Produção Vegetal, End.: R. Brasília, 700, Bloco C, Apto. 303. Bairro Vila Industrial, CEP: 14883-260, Jaboticabal - SP. E-mail: silvabms@ fcav.unesp.br

${ }^{2}$ Bolsista de Produtividade do CNPq - Prof a . Dra. - UNESP/FCAV/DBAA - Departamento de Biologia Aplicada à Agropecuária. E-mail: fabiola@ fcav.unesp.br ${ }^{3}$ Prof. Titular Dr. - UNESP/FCAV/DPV. E-mail: rsader@ fcav.unesp.br

${ }^{4}$ Bolsista de Mestrado da CAPES - Mestranda em Agronomia [Produção e Tecnologia de Sementes]. UNESP/FCAV/DPV. E-mail: nnaomik@fcav.unesp.br 
voltado para baixo (T1); rafe perpendicular e poro germinativo para cima (T2); rafe paralela à superfície e poro germinativo para baixo (T3); rafe paralela à superfície e poro germinativo para cima (T4); as profundidades de semeadura de $0 ; 3$ e $6 \mathrm{~cm}$, com a manutenção das caixas plásticas sob temperatura ambiente de $22,2^{\circ} \mathrm{C} \pm 5,06$ e $70 \%$ de sombreamento artificial (Figura 1).Em dias alternados, por 120 dias, foi anotado o número de plântulas emergidas que apresentavam, pelo menos, $1 \mathrm{~cm}$ do coleóptilo e, em seguida, calculados as porcentagens (PE) e os tempos médios (TM) de emergência, conforme Labouriau \& Agudo (1987), e o índice de velocidade de emergência (IVE), como descrito por Maguire (1962).

O delineamento experimental foi inteiramente casualizado, em esquema fatorial $3 \times 4$, sendo três profundidades e quatro posições de semeaduras, em três repetições de 50 sementes, num total de 12 tratamentos. As médias dos dados percentuais foram transformadas em [arc- sen $(\mathrm{x} / 100)^{0,5}$ ] e comparados pelo teste de Tukey, a 5\% de probabilidade (Pimentel-Gomes, 1987).

O teor de água inicial das sementes foi de $38.6 \% \pm 1,02$, sendo, de acordo com Araújo et al. (1994) e Martins et al. (1999b), superior aos valores críticos e letais para sementes de açaí.

A posição da semente e a profundidade de semeadura agiram de forma independente da porcentagem de emergência de plântulas de açaí. No entanto, considerando o tempo médio e o índice de velocidade de emergência de plântulas, foram observadas interações significativas entre a posição e a profundidade de semeadura.

As posições de semeadura que proporcionaram maiores porcentagens de emergência de plântulas de açaí foram as dos tratamentos T2, T3 e T4, enquanto a menor foi em T1 (Tabela 1). De acordo com Nascimento et al. (2002), foi observada menor porcentagem de emergência de plântulas de Oenocarpus mapora quando as sementes foram semeadas com o poro germinativo voltado para baixo. Entretanto, as diferentes posições de semeadura não afetaram a porcentagem de emergência de plântulas de Euterpe espiritosantensis (Martins et al., 1999a).

$\mathrm{Na}$ profundidade de semeadura de $0 \mathrm{~cm}$, a porcentagem de emergência de plântulas de açaí foi superior e diferiu estatisticamente da observada em $6 \mathrm{~cm}$, sendo que ambas não diferiram da obtida em $3 \mathrm{~cm}$ (Tabela 1). Os menores tempos e maiores índices de velocidade de emergência, independentemente das posições de semeadura, foram observados na profundidade de $0 \mathrm{~cm}$ de semeadura, enquanto valores antagônicos foram encontrados para $6 \mathrm{~cm}$ (Tabelas 2 e 3 ).

$\mathrm{O}$ aumento da barreira física proporcionado pelas camadas mais profundas, em 3 e $6 \mathrm{~cm}$, foi, provavelmente, determinante para o aumento do tempo médio de emergência e, conseqüentemente, para a diminuição do índice de velocidade de emergência (Tabelas 2 e 3). De acordo com o IAC (1974), é recomendável, em viveiro, a semeadura em profundidade de $2 \mathrm{~cm}$ para Euterpe edulis Mart.

Os menores tempos e maiores índices de velocidade de emergência, independentemente das profundidades de semeadura, foram observados nas posições de semeadura T2, $\mathrm{T} 3$ e T4, enquanto os menores valores foram encontrados em T1 (Tabelas 2 e 3). Martins et al. (1999a) e Nascimento et al. (2002) verificaram maior demora para a emergência de plântulas de Euterpe espiritosantensis e Oenocarpus mapora quando as sementes foram colocadas com o poro germinativo voltado para baixo.

Em virtude de a germinação de sementes de Euterpe oleracea ser criptocotiledonar hipógea (Pinheiro, 1986) e apresentar o desenvolvimento tipicamente adjacente ligulado (Tomlinson, 1990), a semeadura com o poro germinativo voltado para baixo torna a própria semente uma barreira adicional para a emergência das plântulas, pois o coleóptilo precisa contorná-la para aflorar à superfície, aumentando o tempo médio de emergência exigido, diminuindo o índice de velocidade de emergência. As diferentes posições de semeadura resultaram em plântulas com diferentes aspectos (Figura 2), de modo semelhante ao descrito por Nascimento et al. (2002), Martins et al. (1999a) e Belin \& Queiroz (1987) em relação à morfologia de plântulas de O. mapora, E. espiritosantensis e E. edulis, oriundas de sementes em diferentes posições de semeadura. Para Euterpe oleracea, não se trata de plântulas anormais, pois mesmo com as deformações, as plântulas apresentaram todas as estruturas essenciais, de acordo com os critérios estabelecidos por Brasil (1992).

Para a semeadura do açaizeiro, o posicionamento mais adequado das sementes é com a rafe perpendicular ao substrato e o poro germinativo voltado para cima. Profundidades iguais ou superiores a $3 \mathrm{~cm}$ são inadequadas para semeadura de Euterpe oleracea Mart. As deformações das plântulas de Euterpe oleracea Mart. causadas pelas diferentes posições de semeadura não as caracterizam como anormais.

TABELA 1 - Porcentagem de emergência de plântula de Euterpe oleracea Mart. em diferentes posições e profundidades de semeadura.

\begin{tabular}{|c|c|c|}
\hline Profundidade (cm)* & Emergência [arc-sen $\left.(\mathrm{x} / 100)^{0,5}\right]$ & Emergência [\%] \\
\hline $\mathbf{0}$ & $68,32 \mathrm{~A}$ & $86,17 \mathrm{~A}$ \\
\hline 3 & $67,59 \mathrm{AB}$ & $85,00 \mathrm{AB}$ \\
\hline 6 & $63,88 \mathrm{~B}$ & $81,50 \mathrm{~B}$ \\
\hline Média & 66,60 & 84,22 \\
\hline Tukey 5\% & \multicolumn{2}{|l|}{4.3461} \\
\hline Posição ${ }^{*^{0}}$ & Emergência [arc-sen $\left.(\mathrm{x} / 100)^{0,5}\right]$ & Emergência [\%] \\
\hline T1 & $63,41 \mathrm{~B}$ & 79,11 \\
\hline T2 & $65,94 \mathrm{AB}$ & 85,33 \\
\hline T3 & $70,30 \mathrm{~A}$ & 88,22 \\
\hline T4 & $66,74 \mathrm{AB}$ & 84,22 \\
\hline Média & 65,33 & 84,22 \\
\hline Tukey 5\% & \multirow{2}{*}{\multicolumn{2}{|c|}{$\begin{array}{l}5,5445 \\
6,4043\end{array}$}} \\
\hline Coeficiente de Variação (\%) & & \\
\hline
\end{tabular}

${ }^{0}$ Poro germinativo voltado para baixo (T1). Poro germinativo voltado para cima - T2. Eixo embrionário paralelo ao substrato com a rafe voltada para baixo - T3. Eixo embrionário paralelo ao substrato com a rafe voltada para cima - T4.

* Médias seguidas de letras iguais, nas colunas, não diferem entre si, pelo teste de Tukey a $5 \%$ de probabilidade. 
TABELA 2 - Desdobramento da interação entre posição e profundidade de semeadura para o tempo médio de emergência de plântulas de Euterpe oleracea Mart.

\begin{tabular}{|c|c|c|c|c|}
\hline \multirow{2}{*}{$\begin{array}{c}\text { Fatores } \\
\text { Profundidade }(\mathrm{cm})^{*}\end{array}$} & \multicolumn{4}{|c|}{ Posições**0 } \\
\hline & T1 & T2 & T3 & T4 \\
\hline 0 & $50,23 \mathrm{Ca}$ & $37,23 \mathrm{Cc}$ & $44,30 \mathrm{Cb}$ & $43,88 \mathrm{Cb}$ \\
\hline 3 & $69,54 \mathrm{Ba}$ & $61,25 \mathrm{Bb}$ & $63,83 \mathrm{Bab}$ & $61,02 \mathrm{Bb}$ \\
\hline 6 & $86,46 \mathrm{Aa}$ & $70,77 \mathrm{Ab}$ & $70,82 \mathrm{Ab}$ & $70,17 \mathrm{Ab}$ \\
\hline Tukey 5\% - Profundidade & \multicolumn{4}{|c|}{5,2764} \\
\hline Tukey 5\% - Posição & \multicolumn{4}{|c|}{5,8294} \\
\hline CV (\%) & \multicolumn{4}{|c|}{4,2586} \\
\hline
\end{tabular}

${ }^{0}$ Poro germinativo voltado para baixo - T1. Poro germinativo voltado para cima - T2. Eixo embrionário paralelo ao substrato com a rafe voltada para baixo - T3. Eixo embrionário paralelo ao substrato com a rafe voltada para cima - T4.

* Médias seguidas de letras maiúsculas iguais, nas colunas, não diferem entre si, pelo teste de Tukey, a 5\% de probabilidade.

** Médias seguidas de letras minúsculas iguais, nas linhas, não diferem entre si, pelo teste de Tukey, a $1 \%$ de probabilidade.

TABELA 3 - Desdobramento da interação entre posição e profundidade de semeadura para o índice de velocidade de emergência de plântulas de Euterpe oleracea Mart.

\begin{tabular}{|c|c|c|c|c|}
\hline \multirow{2}{*}{$\begin{array}{c}\text { Fatores } \\
\text { Profundidade }(\mathrm{cm})^{*}\end{array}$} & \multicolumn{4}{|c|}{ Posições $* * 0$} \\
\hline & T1 & T2 & T3 & T4 \\
\hline 0 & $0,8457 \mathrm{Ac}$ & $1,1977 \mathrm{Aa}$ & $0,9893 \mathrm{Ab}$ & $1,0163 \mathrm{Ab}$ \\
\hline 3 & $0,6190 \mathrm{Ba}$ & $0,6817 \mathrm{Ba}$ & $0,7137 \mathrm{Ba}$ & $0,6810 \mathrm{Ba}$ \\
\hline 6 & $0,3973 \mathrm{Cb}$ & $0,5293 \mathrm{Ca}$ & $0,5463 \mathrm{Ca}$ & $0,5223 \mathrm{Ca}$ \\
\hline Tukey 5\%- Profundidade & \multicolumn{4}{|c|}{0,0927} \\
\hline Tukey 5\% - Posição & \multicolumn{4}{|c|}{0,1025} \\
\hline CV $(\%)$ & \multicolumn{4}{|c|}{6,2483} \\
\hline
\end{tabular}

${ }^{0}$ Poro germinativo voltado para baixo (T1). Poro germinativo voltado para cima (T2). Eixo embrionário paralelo ao substrato com a rafe voltada para baixo (T3). Eixo embrionário paralelo ao substrato com a rafe voltada para cima (T4).

"Médias seguidas de letras maiúsculas iguais, nas colunas, não diferem entre si, pelo teste de Tukey, a 5\% de probabilidade.

** Médias seguidas de letras minúsculas iguais, nas linhas, não diferem entre si, pelo teste de Tukey, a $1 \%$ de probabilidade.
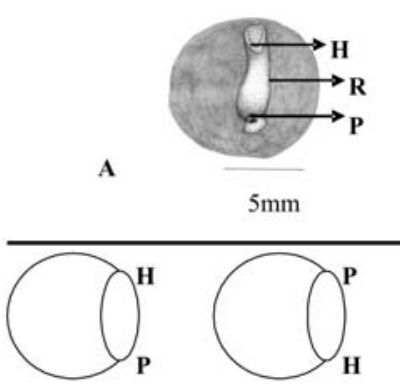

$\mathbf{T}$

C 1

FIGURA 1 - A. Face externa da semente de Euterpe oleracea Mart.. B. Corte longitudinal da semente. C. Esquema das posições de semeadura. PO. Poro germinativo. H. poro germinativo. R. rafe. PO. pseudo-opérculo. E. embrião. EM. endosperma ruminado. T. tegumento.

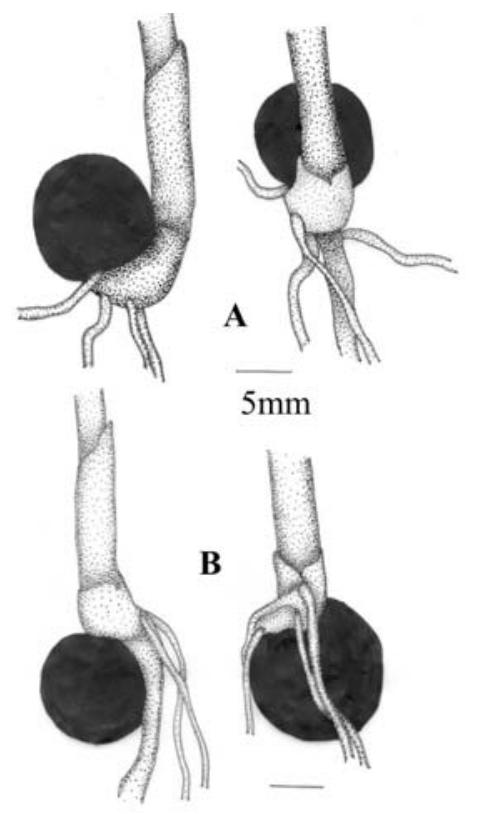

$5 \mathrm{~mm}$
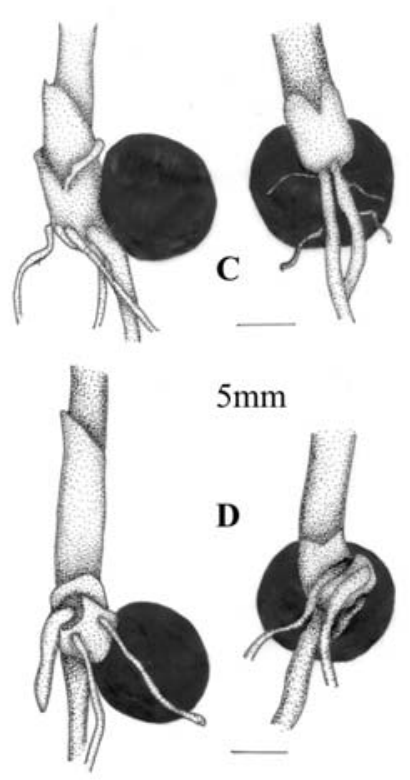

$5 \mathrm{~mm}$
FIGURA 2 - Aspectos morfológicos de plântulas de Euterpe oleracea Mart. semeadas em diferentes posições. A. Poro germinativo voltado para baixo - T1. B. Poro germinativo voltado para cima - T2. C. Eixo embrionário paralelo ao substrato com a rafe voltada para cima - T4. D. Eixo embrionário paralelo ao substrato com a rafe voltada para baixo $-\mathrm{T} 3$.

\section{REFERÊNCIAS}

ARAÚJO, E.F.; SILVA, R.F.; ARAÚJO, R.F. Avaliação da qualidade de sementes de Açaí armazenadas em diferentes embalagens e ambientes. Revista Brasileira de Sementes, Pelotas, v. 16, n. 1, p. 76-79, 1994.

BELIN, M.; QUEIROZ, M.H. Contribuição ao estudo ontogênico das palmeiras. Alguns aspectos morfológicos da germinação de Euterpe edulis Mart. In: ENCONTRO NACIONAL DE PEQUISADORES EM PALMITO, 1., Curitiba, 1987. Resumos...Curitiba: EMBRAPA-CNPF, 1987. p. 211-213. (Documentos, 19).

BRASIL. Ministério da Agricultura e Reforma Agrária. Regras para análise de sementes. Brasília : LAVARV/ SNAD, 1992. 365 p.

CUNHA, A.C.C.; JARDIM, M.A.J. Avaliação do potencial germinativo em Açaí (Euterpe oleracea Mart.) variedades preto, branco e espada. Boletim do Museu Paraense Emílio Goeldi, Série Botânica, Belém, v. 11, n1, p. 55-60, 1995.

DONADIO, L.C.; MÔRO, F.V.; SERVIDONE, A.A. Frutas brasileiras. 2. ed. Jaboticabal: Editora Novos Talentos, 2004. 248p.

IAC - Instituto Agronômico de Campinas. Estudos sobre a cultura do palmiteiro. Boletim Informativo do IAC, Campinas, v. 16, n. 1, p. $1-18,1974$. 
JELLER, H.; PEREZ, S.C.J.G.A. Efeito da salinidade e semeadura em diferentes profundidades na viabilidade e no vigor de Copaifera langsdorffii Desf. - Caesalpiniaceae. Revista Brasileira de Sementes, Pelotas, v. 19, n. 2, p. 218-224. 1997.

LABORIAU, L. G.; AGUDO, M. On the physiology of seed germination in Salvia hispanica L. I. Temperature Effects. Anais da Academia Brasileira de Ciências, Rio de Janeiro, n. 59, p. 37-56. 1987.

MAGUIRE, J.D. Speed of germination: aid in selection and evaluation for seedling emergence and vigor. Crop Science, Madison, v.2, n.2, p.176-177, 1962.

MARCOS-FILHO, J. Fisiologia de sementes de plantas cultivadas. Piracicaba: FEALQ, 2005. 495p.

MARTINS, C.C.; NAKAGAWA, J.; BOVI, M.L.A. Efeito da posição da semente no substrato e no crescimento inicial das plântulas de Palmito-Vermelho (Euterpe espiritosantensis Fernandes - Palmae). Revista Brasileira de Sementes, Pelotas, v. 21, n. 1, p. 164-173, 1999a.

MARTINS, C.C.; NAKAGAWA, J.; BOVI, M.L.A.; STANGUERLIM, H. Teores de água crítico e letal para sementes de Açaí (Euterpe oleracea Mart. - Palmae). Revista Brasileira de Sementes, Pelotas, v. 21, n. 1, p. 125-132, 1999 b.

NAPIER, I. Tecnicas de viveros florestales con referencia especial a centroamerica. Costa Rica, Signa Tepec: Ed. Espemacifor, 1985.274p.

NASCIMENTO, W.M.O.; OLIVEIRA, M.S.P.; CARVALHO, J.E.U.; MÜLLER, C.H. Influência da posição de semeadura na germinação, vigor e crescimento de plântulas de bacabinha (Oenocarpus mapora karsten - Arecaceae). Revista Brasileira de Sementes, Pelotas, v. 24, n. 1, p. 179-182, 2002.

OLIVEIRA, M.S.P.; FERNANDES.G.L.C. Repetibilidade de caracteres de cachos de açaizeiro nas condições de Belém PA. Revista Brasileira de Fruticultura. Jaboticabal, v. 23, n. 3, p. 613-616, 2001.

PIMENTEL-GOMES, F. Curso de estatística experimental. São Paulo: Nobel, 1987.467p.

PINHEIRO, C.U.B. Germinação de sementes de palmeiras: revisão bibliográfica. Teresina - PB: EMBRAPA/UEPAE , 1986. 102p.

TOMLINSON, P.B. The structural biology of palms. Oxford: Clarendon Press, 1990. 460p. 